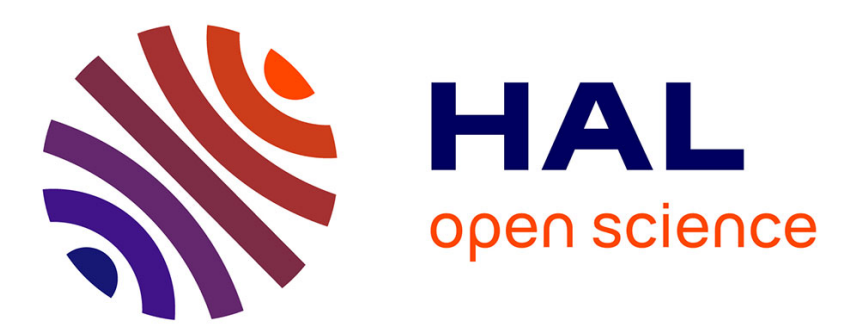

\title{
Further Remarks on Stabilizing Chains of Integrators by Using Network Delays
}

César Fernando Méndez Barrios, Wim Michiels, Silviu-Iulian Niculescu

\section{To cite this version:}

César Fernando Méndez Barrios, Wim Michiels, Silviu-Iulian Niculescu. Further Remarks on Stabilizing Chains of Integrators by Using Network Delays. Proceeding of the 2009 European Control Conference, 2009, http://www.conferences.hu/ecc09/. hal-00450092

\section{HAL Id: hal-00450092 https://hal.science/hal-00450092}

Submitted on 25 Jan 2010

HAL is a multi-disciplinary open access archive for the deposit and dissemination of scientific research documents, whether they are published or not. The documents may come from teaching and research institutions in France or abroad, or from public or private research centers.
L'archive ouverte pluridisciplinaire HAL, est destinée au dépôt et à la diffusion de documents scientifiques de niveau recherche, publiés ou non, émanant des établissements d'enseignement et de recherche français ou étrangers, des laboratoires publics ou privés. 


\title{
Further Remarks on Stabilizing Chains of Integrators by Using Network Delays
}

\author{
César Méndez-Barrios, Wim Michiels and Silviu-Iulian Niculescu
}

\begin{abstract}
This paper focuses on the closed-loop stability of a chain of integrators in a networked-control setting. More precisely, we are interested in using the network-induced delays as control parameters. Similar to the continuous-time case, we will see that a single delay is not sufficient to stabilize a chain having $n$ integrators, but that $n$ delay blocks are able to stabilize such a chain without being able to guarantee an arbitrary pole placement for the corresponding closed-loop system. Several illustrative examples complete the presentation.
\end{abstract}

\section{INTRODUCTION}

In continuous-time, it is well known that the presence of a delay in the feedback loop is often accompanied with "bad" behaviors (oscillations and instability, bandwidth sensitivity, to cite only a few), see, for instance, [7], [20]. However, there exist some cases when the delay may improve systems' stability and the classical example [1] is represented by the an oscillator controlled by one delay "block": (gain, delay), with positive gains and extremely small delay values. Such a property opened an interesting perspective in using delays as control parameters [23], [14] (multiple delays), [19] (bounded input, single delay). Hence, as discussed by [20], the approach can be quite conservative in some situations. Independent, but however correlated to the idea above, the implementation of derivative laws can be done by using delays in order to approximate the derivative, as discussed by [15] and different approaches for the output feedback stabilization problem of a linear system by avoiding output derivatives in the feedback loop have been reported in the literature: [10], [26], [20], to cite only a few.

The aim of this paper is to explore such ideas in the NCS framework, that is to stabilize a chain of integrators by taking into account the network-induced delays and the corresponding sampling period. Although it sounds extremely simple, to the best of the authors' knowledge, such a problem was not fully considered in the literature. Zhang et al. [29] treated the case of a single integrator with one delay block and they derived the stability regions in the parameter-space defined by the delay and the sampling and, for higher-order systems, they suggested the use of simulations in order to approach a solution for the corresponding stabilization problem. It is well known that, the stability and the performances of

César Méndez-Barrios is with L2S (UMR CNRS 8506), CNRSSupélec, 3, rue Joliot Curie, 91192, Gif-sur-Yvette, France. mendez@lss.supelec.fr; (Supported by CONACyT, Mexico).

Wim Michiels is with K.U. Leuven, Department of Computer Science, B3001 Heverlee, Belgium. Wim. Michiels a cs . kuleuven. ac . be.

S.-I. Niculescu is with L2S (UMR CNRS 8506), CNRSSupélec, 3, rue Joliot Curie, 91192, Gif-sur-Yvette, France. Silviu.Niculescu@lss. supelec.fr; Corresponding author.
NCS are affected by the network delays as pointed out by [29], [27]. To overcome such a problem, several approaches have been proposed and, among them: a modelbased method [22] for stability analysis or some optimal controllers when the network-induced delay is smaller [24] or longer [9] than the sampling period, or finally, a queuing mechanism [5] used to reshape random NCS delays to deterministic leading to a time-invariant NCS.

In this context, we are interested in deriving closed-loop stability conditions by using the network-induced delays as control parameters for the continuous process $H_{y u}(s)=1 / s^{n}$ $(n \geq 1)$. The corresponding discrete control law is given by:

$$
u\left(t^{+}\right)=-\sum_{\mu=1}^{m} k_{\mu} y\left(t-\tau_{\mu}\right) \quad t \in\left\{i h+\tau_{m}\right\},
$$

where $i$ is a nonnegative integer and the network-induced delays $\tau_{1}<\tau_{2}<\ldots<\tau_{m}$ are positive real numbers. First, considering a small gain values in the control law we will see that, similarly to the continuous-time case [23], [14] one delay block (gain, delay) cannot stabilize a chain of $n$ integrators, with $n \geq 2$. The approach is based on the use of the complete regular splitting (CRS) property (see, e.g. [16] and Section II for some prerequisites). Next, we will explore the cases when multiple delay blocks are able to stabilize the corresponding chain of integrators. We will see (Section IV) that the closed-loop stability can be obtained by using $n$ delay blocks, but an arbitrary pole placement requires $(n+1)$ delay blocks. In both cases, the corresponding control law is explicitly derived. In the first case ( $n$ delay blocks), the proposed controller leads to some appropriate closedloop characteristic lacunary polynomials (see, e.g. [18]) with nice properties: (a) only one tuning parameter (for improving eventually other performances in closed-loop), (b) particular behaviors of the roots wrt the variations of the corresponding parameter. Several illustrative examples (Sections III-V) complete the presentation.

Throughout the paper the following notation will be adopted: $\mathbb{C}$ is the set of complex numbers, $j=\sqrt{-1}$. For $z \in \mathbb{C}, \angle z \in[0,2 \pi), \mathfrak{R}(z)(\mathfrak{I}(z))$ : argument, real (imaginary) part of $z$. Bold case letter denotes a constant or function vector (matrix). If $A \in \mathbb{C}^{m \times n}$, we write $A=\left[a_{\mu v}\right]_{\mu, v=1}^{m, n}$. If $m=n, A=\left[a_{\mu v}\right]_{\mu, v=1}^{n}$ and its trace is denoted by $\operatorname{Tr}(A)$. For a vector $x \in \mathbb{C}^{n}: x=\left[x_{\mu}\right]_{\mu=1}^{n}$. Let $x, y \in \mathbb{C}^{n}$, the scalar product will be denoted by $\langle x, y\rangle=y^{*} x$, where $y^{*}$ is the complex conjugate transpose of $y$. Finally, the binomial coefficient is defined by $\left(\begin{array}{l}n \\ r\end{array}\right) \triangleq \frac{n !}{r !(n-r) !}$. 


\section{Preliminary Results}

Let $\lambda=\lambda_{0}$ be an isolated eigenvalue of some analyticmatrix-value function $L\left(\lambda, \alpha_{0}\right)$ with partial multiplicities $m_{1} \geq \cdots \geq m_{N}$. Then [8], there exists a neighborhood $\mathscr{O}$ of $\bar{\lambda}=\lambda_{0}$ such that the spectrum of $L(\lambda, \alpha)$ in $\mathscr{O}$ for all complex $\alpha$ sufficiently close to the origin consists of exactly $M:=m_{1}+\cdots+m_{N}$ eigenvalues $\lambda_{i}(\alpha), i=1, \ldots, M$. Furthermore, $\lambda_{k}(\alpha)$ are algebraic functions of $\alpha$ and can be expressed by all the branches of several Puiseux series [4], [8]:

$$
\mu_{v}(\alpha)=c_{v} \alpha^{\frac{1}{q_{v}}}+o\left(|\alpha|^{\frac{1}{q_{v}}}\right), \quad v=1, \ldots, N^{\prime}, q_{v} \in \mathbb{N},
$$

where $q_{1} \geq \cdots \geq q_{N^{\prime}}$ and $q_{1}+\cdots+q_{N^{\prime}}=M$. A completely regular splitting (CRS) [16] property of the eigenvalue $\tilde{\lambda}=$ $\lambda_{0}$ at $\alpha=0$ corresponds to: $N=N^{\prime}, q_{i}=m_{i}$ and $c_{i} \neq 0$, $i=1, \ldots, N$. The following result characterizes the CRS property:

Theorem 1 ([8]): With the notations above, let $\lambda=0$ be an eigenvalue of $L(\lambda, 0)$ of geometric multiplicity $N$ and algebraic multiplicity $M$. Suppose also that for every generating eigenvector $x$ of $L(\lambda, 0)$ there exists a generating eigenvector $\hat{x}$ of $(L(\lambda, 0))^{*}$ such that

$$
\left\langle\frac{\partial L}{\partial \alpha}(0,0) x, \hat{x}\right\rangle \neq 0
$$

Then the eigenvalue $\lambda=0$ possesses the CRS property.

\section{PROBlem FORMUlation AND SOME EXAMPLES}

Consider the following chain of integrators system,

$$
y^{(n)}(t)=u\left(t^{+}\right) \quad t \in\left[i h+\tau_{m},(i+1) h+\tau_{m}\right)
$$

where $\tau=: \tau_{1}<\tau_{2}<\ldots<\tau_{m}$ and $u\left(t^{+}\right)$is given by

$$
u\left(t^{+}\right)=-\sum_{\mu=1}^{m} k_{\mu} y\left(t-\tau_{\mu}\right), \quad t \in\left\{i h+\tau_{m}, i \in \mathbb{N}\right\}
$$

\section{A. Discretized Delay Case and Scaling Properties}

Let $\tilde{\tau}$ be the induced network delay, $h$ the sampling period, and let $\tau_{\mu}$ for $\mu=\overline{1, m}$ such that, $\tau_{1}<\ldots<\tau_{m}$. Then, after some algebraic manipulation the discretized system can write as [2]:

$$
y[i+1]=\Phi(h) y[i]+\Delta_{0}(k) u[i-d+1]+\Delta_{1}(k) u[i-d],
$$

where $d \in \mathbb{N}$ and

$$
\begin{aligned}
\Phi(h) \triangleq & {\left[\phi_{\mu v}(h)\right]_{\mu, v=1}^{n} } \\
& \text { with } \phi_{\mu v}(h) \triangleq\left\{\begin{array}{ccc}
\frac{h^{v-\mu}}{(v-\mu) !} & \text { if } \quad & v \geq \mu \\
0 & \text { if } & v<\mu
\end{array}\right. \\
\Delta_{0}(k ; m) \triangleq & \sum_{\mu=1}^{m} \Gamma\left(0, h-\tau_{\mu}\right) k_{\mu} \\
\Delta_{1}(k ; m) \triangleq & \sum_{\mu=1}^{m} \Gamma\left(h-\tau_{\mu}, h\right) k_{\mu} \\
\Gamma\left(t_{i}, t_{f}\right) \triangleq & {\left[\sigma_{\mu}\left(t_{i}, t_{f}\right)\right]_{\mu=1}^{n} } \\
& \quad \text { with } \sigma_{\mu}\left(t_{i}, t_{f}\right) \triangleq \frac{\left(t_{f}\right)^{n-\mu+1}-\left(t_{i}\right)^{n-\mu+1}}{(n-\mu+1) !}
\end{aligned}
$$

Define now the augmented state vector as $z[i] \triangleq$ $\left[y^{T}[k], u[i-d], u[i-d+1], \ldots, u[i-1]\right]^{T}$, leading to the augmented closed-loop system:

$$
z[i+1]=\tilde{\Phi}(h, \tau, k) z[i]
$$

Equation (5) describes a general situation, that is, when the induced network delay is larger/smaller than the sampling period. Under these observations, we have:

Remark 1 (Smaller delay): Let $\tau$ be the induced network delay, such that $\tau=: \tau_{1}<\cdots<\tau_{m}<h$. Then, $d=1$ in (5) and the transfer matrix of the augmented closed-loop system rewrites as:

$$
\tilde{\Phi}(h, \tau, k) \triangleq\left[\begin{array}{cc}
\Phi(h)-\Delta_{0}(k ; m) e_{1}^{T} & \Delta_{1}(k ; m) \\
-e_{1}^{T} & 0
\end{array}\right] .
$$

Remark 2 (Larger delay): Let $\tilde{\tau}$ be the induced network delay and $h$ the sampling period, such that $\tilde{\tau}=(d-1) h+\tau$ and $h>\tau$. Then, $\tau=: \tau_{1}<\cdots<\tau_{m}<h$, and the corresponding transfer matrix becomes,

$$
\tilde{\Phi}(h, \tau, k) \triangleq\left[\begin{array}{ccccc}
\Phi(h) & \Delta_{1}(k ; m) & \Delta_{0}(k ; m) & \cdots & 0 \\
0 & 0 & 1 & \cdots & 0 \\
\vdots & \vdots & \vdots & \ddots & \vdots \\
0 & 0 & 0 & \cdots & 1 \\
-e_{1}^{T} & 0 & 0 & \ldots & 0
\end{array}\right]
$$

As in the continuous case, we have the following property.

Property 1: The control law

$$
u\left(t^{+}\right)=-\sum_{\mu=1}^{m} k_{\mu} y\left(t-\tau_{\mu}\right), \quad t \in\left[i h+\tau_{m},(i+1) h+\tau_{m}\right)
$$

is asymptotically stabilizing if and only if the control law

$$
u\left(t^{+}\right)=-\sum_{\mu=1}^{m} \frac{k_{\mu}}{\rho^{n}} y\left(t-\rho \tau_{\mu}\right) \quad t \in \alpha\left[i h+\tau_{m},(i+1) h+\tau_{m}\right),
$$

with $\rho>0$, is stabilizing.

Proof: This property can be show by taking $z_{i} \triangleq \rho^{n-i} x_{i}$, $\tilde{t} \triangleq \rho t$ and $\tilde{u^{+}} \triangleq \frac{1}{\rho^{n}} \sum_{i=1}^{m} z\left(\tilde{t}-\rho \tau_{i}\right)$, showing the equivalence of the systems.

\section{B. Motivating Examples}

In the scalar case, [29] derived the stability region in the $(h(k), \tau)$ parameter space for the case of one integrator. The corresponding NCS will be stable if and only if:

$$
\max \left\{\frac{1}{2} h-\frac{1}{k}, 0\right\}<\tau<\min \left\{\frac{1}{k}, h\right\}
$$

Remark 3: It is easy to see from inequality (9) how the scaling property works.

Proposition 1: If $n>2$, then the closed-loop system consisting of a chain of $n$ integrators

$$
y^{(n)}(t)=u(t), \quad t \in[i h+\tau,(i+1) h), \quad \tau<h
$$

and a control law of the form

$$
u\left(t^{+}\right)=-k y(t-\tau), \quad t \in\{i h+\tau \mid i \in \mathbb{N}\}
$$

is unstable for small values of the controller gain $k$. 
Proof: The assertion follows from the behavior of the eigenvalue $\lambda_{0}=1$ for $k=0$ as $|k|$ is increased from zero, and is based on Theorem 1. To this end, consider

$L(\tilde{\lambda}, k)=\left[\begin{array}{cc}\Phi(h)-I-\tilde{\lambda} I & 0 \\ -e_{1}^{T} & -\tilde{\lambda}-1\end{array}\right]+\left[\begin{array}{cc}-\Delta_{0}(k ; 1) e_{1}^{T} & \Delta_{1}(k ; 1) \\ 0 & 0\end{array}\right]$

where $\tilde{\lambda}:=\lambda-1$. From the definition of $\Phi(h)$ it follows that the algebraic and geometric multiplicity of the eigenvalue $\tilde{\lambda}=0$ for $k=0$ is $n$ and 1 , respectively. Furthermore the right and left eigenvectors are given by $x=e_{1}$ and $\hat{x}=e_{n}$. Next, we have

$$
\frac{\partial L}{\partial k}(\lambda, k)=\left[\begin{array}{cc}
-\Gamma(0, h-\tau) & \Gamma(h-\tau, h) \\
0 & 0
\end{array}\right] .
$$

It is easy to see that (2) holds if and only if the equation

$$
\begin{aligned}
L(0,0) y & =\frac{\partial L}{\partial k}(0,0) e_{1} \\
\Leftrightarrow\left[\begin{array}{cc}
\Phi(h)-I & 0 \\
-e_{1}^{T} & -1
\end{array}\right] y & =-\left[\begin{array}{c}
\frac{(h-\tau)^{n}}{n !} \\
\vdots \\
\frac{h-\tau}{1 !} \\
0
\end{array}\right]
\end{aligned}
$$

has no solution for $y$. Since, by hypothesis, $h>\tau$, this is the case and the eigenvalue $\tilde{\lambda}=0$ has the CRS property. It follows that for small $|k|$ it can be expanded as

$$
\tilde{\lambda}_{i}(k)=c e^{j \frac{2 \pi i}{n}} k^{\frac{1}{m}}+o\left(k^{\frac{1}{m}}\right), i=1, \ldots, n,
$$

for some $c \in \mathbb{C}$. Since $\lambda=1+\tilde{\lambda}$ it follows that the original system always has one eigenvalue outside the unit circle for small values of $|k|$.

Example 1: Consider first the double integrator. As mentioned above, it cannot be stabilized by using a single blockdelay (see Fig.1 (upper)). However, the use of a controller involving two delays will be able to stabilize it. Indeed, consider $\tau_{1}:=\tau<\frac{h}{3}, \tau_{2}:=\tau+\varepsilon$ with $0<\varepsilon<\frac{2}{3}(h-3 \tau)$. Then the control gains:

$$
\begin{aligned}
& k_{1}(h, \tau, \varepsilon) \triangleq \frac{6 h^{2}+4 h(\tau+\varepsilon)-6(\tau+\varepsilon)^{2}}{h \varepsilon\left(4 h^{2}+2 \tau(\tau+\varepsilon)-3 h(2 \tau+\varepsilon)\right.} \\
& k_{2}(h, \tau, \varepsilon) \triangleq \frac{-6 h^{2}-4 h \tau+6 \tau^{2}}{h \varepsilon\left(4 h^{2}+2 \tau(\tau+\varepsilon)-3 h(2 \tau+\varepsilon)\right.}
\end{aligned}
$$

will define a stabilizing control law (4).

Consider now the general case. The characteristic polynomial of $\tilde{\Phi}(h, \tau, k)$

$$
P_{c l}(z):=z^{n+d}+a_{n+d-1}(h, \tau, k) z^{n+d-1}+\cdots+a_{0}(h, \tau, k)
$$

describes the general case for the augmented closed-loop system (6). However, in order to simplify our analysis we will consider in the sequel the smaller delays case (i.e., $d=1$ ).

Assertion 1: The coefficients of $P_{c l}(z)$ satisfy the following properties

1) $a_{\mu}(h, \tau, k)$ are affine functions in $k\left(\beta_{\mu} \in \mathbb{R}\right)$ :

$$
a_{\mu}(h, \tau, k)=\sum_{v=1}^{m} k_{v} \alpha_{\mu, v}\left(h, \tau_{v}\right)+\beta_{\mu}
$$

2) $\alpha_{\mu, v}(h, \tau)$ is a polynomial function in $(h, \tau)$ satisfying $\alpha_{\mu, v_{i}}(h, \tau)=\alpha_{\mu, v_{j}}(h, \tau) \quad$ for $\quad i \neq j$.

This assertion follows by a straightforward application of the Laplace expansion's [11] to the last row of:

$$
\tilde{\Phi}_{c l}(z, h, \tau, k):=\left[\begin{array}{cc}
z I_{n \times n}+\Delta_{0}(k) e_{1}^{T}-\Phi(h) & -\Delta_{1}(k) \\
e_{1}^{T} & z
\end{array}\right]
$$

Remark 4: In order to calculate $\alpha_{\mu, v_{i}}\left(h, \tau_{v_{i}}\right)$, it is sufficient to take $m=n=1$ (see Assertion 1).

Remark 5: By applying the determinant properties [11], it is easy to see that $\alpha_{n, v}(h, \tau), \beta_{n}$ and $\alpha_{0, v}(h, \tau), \beta_{0}$ satisfy $\alpha_{n, v}(h, \tau)=\frac{(h-\tau)^{n}}{n !}, \beta_{n}=-n, \quad \alpha_{0, v}(h, \tau)=\frac{\tau^{n}}{n !}, \beta_{0}=0$.

Remark 6: Let $z^{(0)}:=\left\{z_{1}^{(0)}, \ldots, z_{n+1}^{(0)}\right\}$ be the set of roots of $P_{c l}$. Then, the fact that the uncontrolled system has $n+1$ roots on $\mathscr{C}(0,1)$ simply points out that if $m=n$ in the control law (4) we will not have enough "degrees-of-freedom"for an arbitrary pole placement.

Example 2: Consider now the triple integrator:

$$
y^{(3)}(t)=u\left(t^{+}\right), \quad t \in[i h+\tau,(i+1) h+\tau), \quad \tau<h .
$$

Taking $m=3$ in (4), denoting the roots of $P_{c l}$ by $\left\{\zeta_{\mu}\right\}$ for $\mu=\{\overline{1,4}\}$ and considering $\zeta_{4}\left(\zeta_{1}, \zeta_{2}, \zeta_{3}\right)$. Then, the
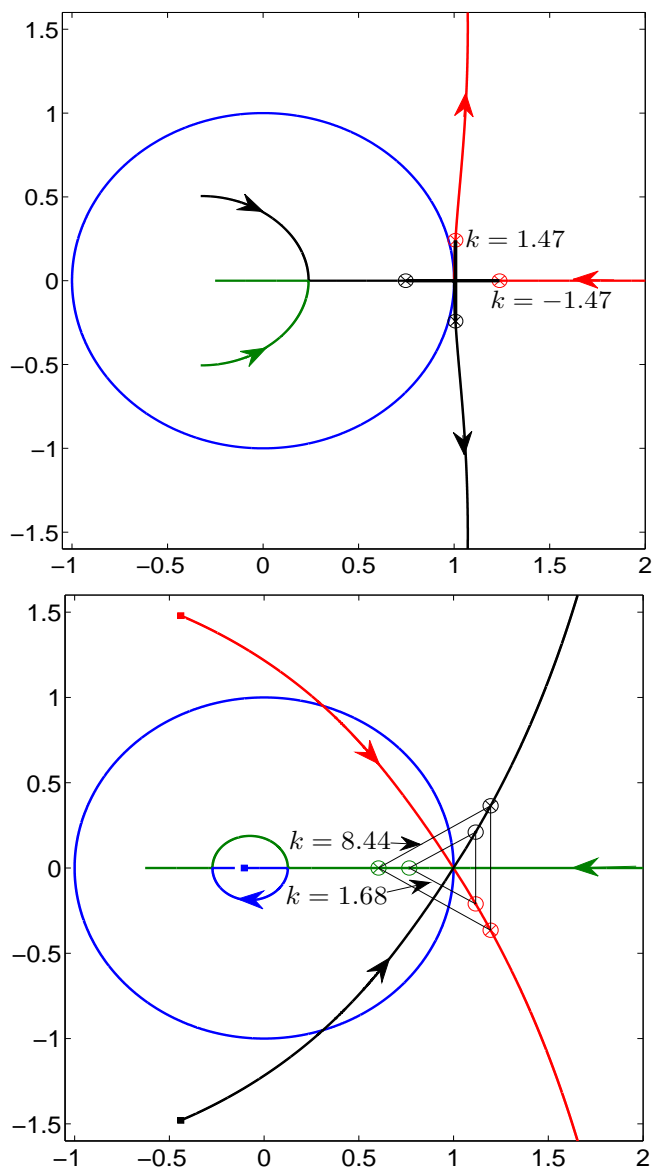

Fig. 1. Completely Regular Splitting property illustrating Proposition 1 for $\lambda=1$. (Upper) Double-integrator $(n=2)$ for $k \in\left[-\frac{2 !}{\tau^{2}}, \frac{2 !}{\tau^{2}}\right]$. (Lower) Triple-integrator $(n=3)$ for $k \in\left[-\frac{3 !}{\tau^{3}}, \frac{3 !}{\tau^{3}}\right]$. 


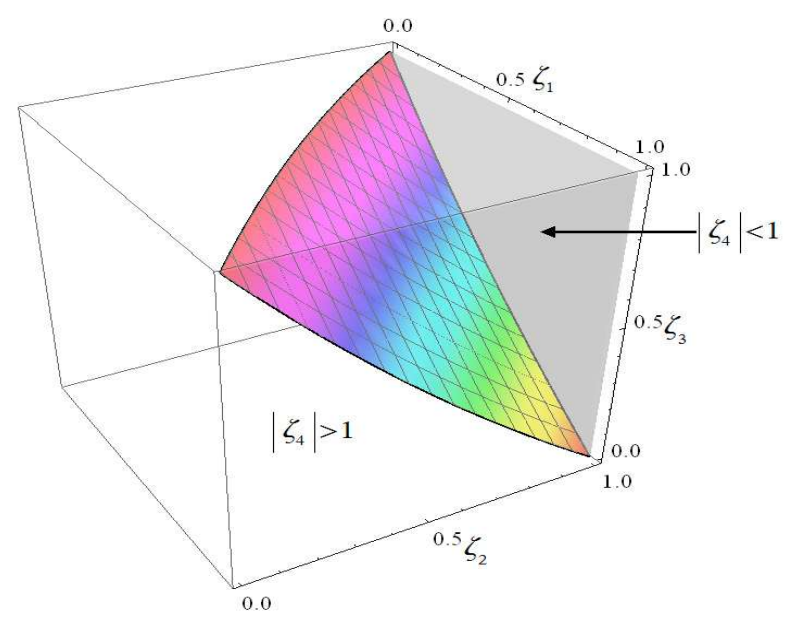

Fig. 2. Admissible pole-placement for the control law (4) with $m=n=3$

only admissible roots $\left|\zeta_{\mu}\right|<1$ for $\mu=\{\overline{1,3}\}$ such that $\left|\zeta_{4}\left(\zeta_{1}, \zeta_{2}, \zeta_{3}\right)\right|<1$ are depicted in figure Fig.2 and such a situation illustrates Remark 6 above.

\section{Problem Formulation}

As briefly discussed in the previous section, we will focus on finding conditions on the $m$ gain parameters $k=$ $\left(k_{1}, \ldots, k_{m}\right)$ such that (4) is a stabilizing control law. In particular, we will consider two cases: $m=n+1$ (exact pole placement) and $m=n$ (called reduced controller).

\section{MAin Results}

\section{A. Control law based on exact pole placement}

Denote the set of desired closed-loop roots by $\lambda^{(0)}:=$ $\left\{\lambda_{1}, \ldots, \lambda_{n+1}\right\}$ and the corresponding characteristic polynomial by:

$$
P_{d}(z, s):=z^{n+1}-c_{1} z^{n}+c_{2} z^{n-1}+\cdots+(-1)^{n+1} c_{n+1}
$$

where $c_{k}$ is the $k^{\text {th }}$ symmetric function of $\lambda^{(0)}$ defined as the sum of the product of the eigenvalues taken $k$ at the time:

$$
c_{k}:=\sum_{1 \leq i_{1} \leq \cdots \leq i_{k} \leq n+1} \lambda_{i_{1}} \cdots \lambda_{i_{k}}
$$

Proposition 2 (Exact pole-placement): Assume that $\tau=$ : $\tau_{1}<\tau_{2}<\ldots<\tau_{n+1}<h$. Under the notations above, define the gain:

$$
k=A^{-1} \tilde{c}^{T}
$$

with $A \triangleq\left[\bar{\alpha}_{\mu-1}\left(h, \tau_{v}\right)\right]_{\mu, v=1}^{n+1}$,

$\tilde{c} \triangleq\left[\begin{array}{llll}(-1)^{n+1} c_{n+1} & (-1)^{n} c_{n}-(-1)^{n}\left(\begin{array}{l}n \\ 0\end{array}\right) & \cdots & -c_{1}+\left(\begin{array}{c}n \\ n-1\end{array}\right)\end{array}\right]$

where $\bar{\alpha}_{i}(h, \tau)$ is defined recursively by taking $B_{0}:=I$ and,

$$
\begin{aligned}
\bar{\alpha}_{\mu}(h, \tau) & \triangleq-\frac{1}{n-\mu+1} \frac{\partial}{\partial k_{v}} \operatorname{Tr}\left(\tilde{\Phi}(h, \tau, k) B_{n-\mu}\right) \\
B_{\mu} & \triangleq-\frac{\operatorname{Tr}\left(\tilde{\Phi}(h, \tau, k) B_{\mu-1}\right)}{\mu} I+\tilde{\Phi}(h, \tau, k) B_{\mu-1}
\end{aligned}
$$

Then the corresponding control law (4) guarantees the closed-loop characteristic roots are located at $\lambda^{(0)}$

Proof: See the appendix.

\section{B. Reduced Controller. Case $m=n$}

We focus now on finding the control law $k=\left(k_{1}, \ldots, k_{n}\right)$ such that the closed-loop characteristic polynomial becomes:

$$
P\left(z ; p, i_{p}\right):=z^{n+1}+p\left(z^{n-i_{p}+1}+z^{n-i_{p}}+\cdots+z+1\right)
$$

with $1 \leq i_{p} \leq n+1$ and $n \geq 1$. It is important to mention that the lacunary polynomials of the form (16) have received some attention in the literature [12] in the context of delaydifference equations. Its main interest lies in interesting properties to be exploited in what follows:

Property 2: The following properties hold for $P\left(z ; p, i_{p}\right)$ :

(i) the moduli of the roots increase as $|p|$ increases.

(ii) the roots are inside the unit circle if $|p|<\frac{1}{n-i_{p}+2}$.

Proof: First, $P\left(z ; p, i_{p}\right) \equiv z^{n+1}+p \frac{z^{n-i_{p}+2}-1}{z-1}$. Next, for (i), see [12]. (ii) Take now $f(z) \triangleq z^{n+1}$ and $g\left(z ; i_{p}\right) \triangleq$ $p\left(\sum_{k=0}^{n-i_{p}+1} z^{k}\right)$. For all $z \in \mathbb{C}$, we have that $\left|g\left(z ; i_{p}\right)\right|=$ $|p|\left|\sum_{k=0}^{n-i_{p}+1} z^{k}\right| \leq|p| \sum_{k=0}^{n-i_{p}+1}\left|z^{k}\right|$. Then, taking $|p|<\frac{1}{n-i_{p}+2}$ for $|z|=1$ we have that $|f(z)|>\left|g\left(z ; i_{p}\right)\right|$. Then, by a straightforward application of Rouche's lemma [18], $P\left(z ; p, i_{p}\right) \equiv$ $f(z)+g\left(z ; i_{p}\right)$ is a Schur-stable polynomial.

Remark 7: The proof above guarantees not only the existence of some "stabilizing" parameter $p$, but it also gives a "cheap" way to compute it.

Define now $\mathscr{X}$ as the set of real zeros of the polynomial:

$$
T_{n+1}(x) \sum_{j=0}^{n-i_{p}} U_{j}(x)-U_{n}(x) \sum_{j=0}^{n-i_{p}+1} T_{j}(x),
$$

and introduce the following quantities:

$p^{-}=\max _{x^{*} \in \mathscr{X}}\left\{\frac{-U_{n}\left(x^{*}\right)}{\sum_{j=0}^{n-i_{p}} U_{j}\left(x^{*}\right)}<0\right\}, p^{+}=\min _{x^{*} \in \mathscr{X}}\left\{-\frac{U_{n}\left(x^{*}\right)}{\sum_{j=0}^{n-i p} U_{j}\left(x^{*}\right)}>0\right\}$.

Proposition 3: The polynomial $P\left(z ; p^{*}, i_{p}\right)$ is Schur stable if and only if

$$
\max \left\{\frac{-1}{n-i_{p}+2}, p^{-}\right\}<p^{*}<p^{+} .
$$

Proof: The polynomial $P\left(z ; 0, i_{p}\right)$ is Schur. Now, since the roots of a polynomial are continuous with respect to their coefficients (see, e.g., [3], [25]), it follows the existence of some real $p$ close to 0 such that $P\left(z ; p, i_{p}\right)$ is still Schur stable. Moreover, in the limit case, there exists a $p^{*} \in[-1,1]$ such that $P\left(z^{(0)} ; p^{*}, i_{p}\right)=0 \Rightarrow z^{(0)}=e^{j \theta}, \theta \in[0,2 \pi)$. Then, $\Re\left(P\left(z^{(0)} ; p^{*}, i_{p}\right)\right)=0$ and $\frac{\mathfrak{I}\left(P\left(z^{(0)} ; p^{*}, i_{p}\right)\right)}{\sin \theta}=0$ lead to:

$$
\begin{aligned}
T_{n+1}(x)+p^{*} \sum_{j=0}^{n-i_{p}+1} T_{j}(x) & =0 \\
U_{n}(x)+p^{*} \sum_{j=0}^{n-i_{p}} U_{j}(x) & =0
\end{aligned}
$$


where $x=\mathfrak{R}\left(z^{(0)}\right)=\cos \theta$. Equations (20)-(21) will give the whole set of solutions, except the singular point $z^{(0)}=1$. In this last case, $p^{*}$ can be obtained by solving $P\left(1 ; p^{*}, i_{p}\right)=0$. Some simple algebraic manipulations lead to the conditions (19).

Remark 8: It follows from the first assertion of Property 2 that the condition (19) defines the whose set of solutions. Notice also that, (17) has at most $n$ solutions. Finally, [12] proposed a different argument for proving a similar property.

Proposition 4: Let $\tau$ be the induced network delay, $\tau_{2}, \ldots, \tau_{n}$ chosen like $\tau_{i}=\tau+(i-1) \varepsilon$ for $i=\{2, \ldots, n\}$ and $p^{*}$ be chosen satisfying (19) for some $1 \leq i_{p} \leq n+1$. Then, the control law (4) with,

$$
k(\varepsilon)=\bar{A}^{-1} p
$$

where $\bar{A} \triangleq\left[\bar{\alpha}_{\mu}\left(h, \tau_{v}\right)\right]_{\mu, v=1}^{n}$,

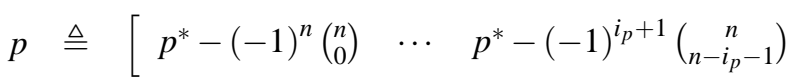

$$
\begin{aligned}
& \left.(-1)^{i_{p}+1}\left(\begin{array}{c}
n \\
n-i_{p}
\end{array}\right) \quad \cdots \quad\left(\begin{array}{c}
n \\
n-1
\end{array}\right)\right]
\end{aligned}
$$

guarantees the closed-loop stability, whenever $\varepsilon$ satisfies,

$$
p_{0}^{-}<\bar{\alpha}_{0}\left(h, \tau_{1}\right) k_{1}(\varepsilon)+\cdots+\bar{\alpha}_{0}\left(h, \tau_{n}\right) k_{n}(\varepsilon)<p_{0}^{+}
$$

for $\varepsilon>0, h>\tau+(n-1) \varepsilon$, and where $p_{0}^{ \pm}$are given by:

$$
\begin{aligned}
& p_{0}^{-} \triangleq \max \left\{p_{0},\left\{\begin{array}{cc}
p^{*}-1 & \text { if } n-i_{p} \in 2 \mathbb{N} \\
-1 & \text { otherwise }
\end{array} \mid p_{0}<p^{*}\right\}\right. \\
& p_{0}^{+} \triangleq \min \left\{p_{0} \mid p_{0}>p^{*}\right\}
\end{aligned}
$$

where $p_{0}$ is the set given by,

$$
p_{0} \triangleq-\left\{T_{n+1}\left(x^{*}\right)+p^{*} \sum_{l=1}^{n-i_{p}+1} T_{l}\left(x^{*}\right)\right\}
$$

and $x^{*}$ is a root of the following polynomial

$$
U_{n}(x)+p^{*} \sum_{l=0}^{n-i_{p}} U_{l}(x)
$$

Proof: According to Proposition 3, for the invertibility of $\bar{A}$ is sufficient to have $\tau_{1} \neq \cdots \neq \tau_{n}$ and $h>\tau_{i}$ for all $i=\overline{1, n}$. Since this fact is fulfilled by hypothesis, $k(\varepsilon)$ is well defined. Then, let $k(\varepsilon)$ be given by (22). It is clear from Proposition 2 that the closed-loop system will be rewritten as follows:

$P_{c l}\left(z ; p^{*}, \tilde{p}^{*}, i_{p}\right)=z^{n+1}+p^{*}\left(z^{n-i_{p}+1}+z^{n-i_{p}}+\cdots+z\right)+\tilde{p}^{*}(\varepsilon)$, where $\tilde{p}^{*}(\varepsilon)$ is given by:

$$
\tilde{p}^{*}(\varepsilon) \triangleq \bar{\alpha}_{0}\left(h, \tau_{1}\right) k_{1}(\varepsilon)+\cdots+\bar{\alpha}_{0}\left(h, \tau_{n}\right) k_{n}(\varepsilon)
$$

Since by assumption, $p^{*}$ satisfies (19), we have that, for $\tilde{p}^{*}(\varepsilon)=p^{*}$, the closed-loop system is asymptotically stable. Then, similarly to the proof of Proposition 3, there exists some interval $\left(p_{0}^{-}, p_{0}^{+}\right)$including $p^{*}$ such that the system remains asymptotically stable. In the limit case, $P_{c l}\left(e^{j \theta} ; p^{*}, \tilde{p}^{*}\right)=0$. Taking the corresponding real and imaginary parts $\left(\Re\left[P_{c l}\left(e^{j \theta} ; p^{*}, \tilde{p}^{*}\right)\right]=0, \mathfrak{I}\left[P_{c l}\left(e^{j \theta} ; p^{*}, \tilde{p^{*}}\right)\right]=0\right)$ and using the Chebyshev polynomials, we obtain (25)-(26), respectively. Equation (25) gives the set of all possible intervals including $p^{*}$, excepting for the singular point $\theta=$ $\pi$. At this point, we must have $p_{0}^{-}=p *-1$ whenever $n-i_{p} \in 2 \mathbb{N}$. Then, in order to preserve the stability, we must choose the smallest interval, i.e., $p_{0}$ must be contained in the interval $\left(p_{0}^{-}, p_{0}^{+}\right)$given by equation (24). This means that, if $p_{0}^{-}<\tilde{p^{*}}(\varepsilon)<p_{0}^{+}$the closed-loop system will be asymptotically stable. Since this is equivalent with equation (23), the proof is completed.

\section{ILlustrative EXAMPLE}

In order to illustrate how the present methodology works, we consider a fourth-order chain of integrators as:

$$
y^{(4)}(t)=u\left(t^{+}\right), \quad t \in[i h+\tau,(i+1) h), \quad \tau<h
$$

where $\tau=0.1$ is the induced-network delay and $h=0.6$ is the sampling period. Taking $\tau_{i}=\tau+(i-1) \varepsilon$ for $i=$ $\{\overline{2,4}\}$ in the control law (4), then applying Proposition 3-4 we obtain $p \in(-0.25,0.4450)$ and $p_{0} \in(-0.7181,0.8158)$ (where the later interval was obtained by choosing $p^{*}=0.2$ ), respectively. Then, according with Proposition 4, the system (27) is asymptotically stable whenever $\varepsilon \in(0,0.01202)$. In order to illustrate this result graphically, we plot the roots' trajectories for $p_{0} \in(-0.7181,0.8158)$ in Fig.3.

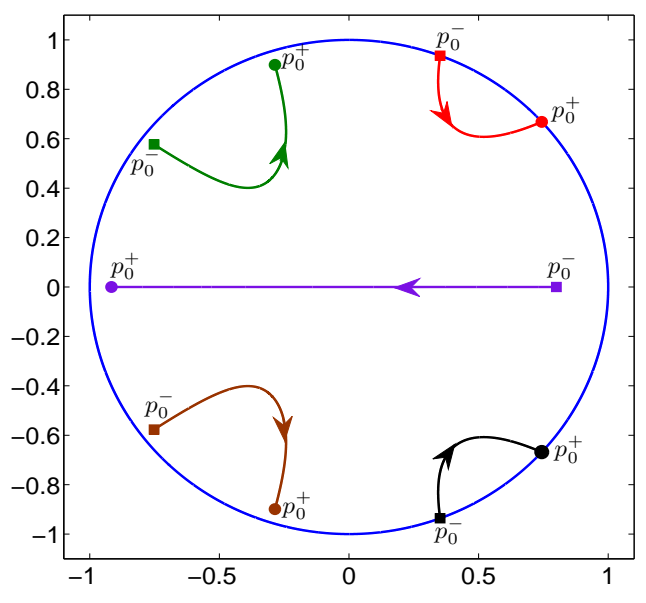

Fig. 3. Root trajectories for $p_{0} \in(-0.7181,0.8158)$

\section{CONCLUDING REMARKS}

In this note, the problem of stabilizing a chain of integrators by using network delays as controller parameters was addressed. Several algorithms and properties have been outlined and various illustrative examples proving the theoretical results have been also proposed. For the sake of brevity, only the case of delays smaller than the sampling period has been considered. However, the approach proposed in the paper applies also to the case of larger delays. 


\section{REFERENCES}

[1] Abdallah, C., Dorato, P., Benitez-Read, J. and Byrne, R.: Delayed positive feedback can stabilize oscillatory systems. Proc. American Contr. Conf. (1993) 3106-3107.

[2] Åström, K.J. and Wittenmark, B.: Computer Controlled Systems: Theory and Design (Prentice Hall, 3rd. Edt., 1997).

[3] Bhattacharyya, S.P., Chapellat, H. and Keel, L.H.: Robust Control: The Parametric Approach (Prentice Hall, 1995).

[4] Bliss, G.A.: Algebraic Functions (Dover, New York; 1966).

[5] Chan, H. and Özgüner, Ü.: Closed-loop control of systems over a communication network with queues, Internatinal Journal of Control, 62:3 (1995) 493-510.

[6] Gao, H., Chen, T. and Lam, J.: A new delay system approach to network-based control, Automatica, 44 (2007) 39-52.

[7] Gu, K., Kharitonov, V.L. and Chen, J.: Stability of time-delay systems (Birkhauser: Boston, 2003).

[8] Hryniv, R. and Lancaster, P.: On the Perturbation of Analytic Matrix Functions, Integral Equations and Operator Theory, 34 (1999) 325338.

[9] Hu, S.S. and Zhu, Q.X.: Stochastic optimal control and analysis stability of networked control systems with long delay, Automatica, 39 (2003) 1877-1884.

[10] Ilchmann, A. and Sangwin, C.J.: Output feedback stabilization of minimum phase systems by delays, System and Control Letters, 52 (2004) 233-245.

[11] Lancaster, P.: Theory of Matrices (Academic Press: New York, 1969).

[12] Kaewong, T., Lenbury, Y. and Niamsup, P.: A note on asymptotic stability conditions for delay difference equations, Int. J. Math. Mathematical Sci. (2005) 1007-1013.

[13] Kato, T. Perturbation Theory of Linear Operators (Springer: Berlin, 1976).

[14] Kharitonov, V.L., Niculescu, S.-I., Moreno J. and Michiels, W.: Static output feedback stabilization: Necessary conditions for multiple delay controllers, IEEE Trans. Aut. Control, 50 (2005) 82-86.

[15] Kokame, H., Hirata, K., Konishi, K. and Mori, T.: Difference feedback can stabilize uncertain steady states, IEEE Trans. on Automatic Control, 46 (2001) 1908-1913.

[16] Langer, H., Najman, B. and Vaselic, K.: Perturbation of the Eigenvalues of Quadratic Matrix Polynomials, SIAM J. Matrix Anal. Appl., 13:2 (1992) 474-489.

[17] Mason, J.C. and Handscomb, D.C.: Chebyshev Polynomials (Chapman and Hall/CRC, 2003).

[18] Marden, M.,: Geometry of Polynomials (AMS, 1989).

[19] Mazenc, F., Mondié, S. and Niculescu, S.-I.: Global asymptotic stabilitation for chains of integrators with a delay in the input, IEEE Trans. Automat. Contr., vol. 48, 2003, pp.57-63.

[20] Michiels, W. and Niculescu, S.-I.: Stability and stabilization of timedelay systems. An eigenvalue approach (SIAM: Philadelphia, USA, 2007).

[21] Meyer, C.D.: Matrix analysis and applied linear algebra (SIAM: Phildelphia, USA, 2000).

[22] Montestruque, L.A. and Antsaklis, P.: On the model-based control of networked systems, Automatica, 39 (2003) 1837-1843.

[23] Niculescu, S.-I. and Michiels, W.: Stabilizing a chain of integrators using multiple delays, IEEE Trans. Aut. Control, 49 (2004) 802-807.

[24] Nilsson, J., Bernhardsson, B. and Wittenmark, B.: Stochastic analysis and control of real time systems with random time delays, Automatica, 34 (1998) 57-64.

[25] Rahman, Q.I. and Schmeisser, G.: Analytic Theory of Polynomials (Oxford Press: Oxford, 2002).

[26] Roy, S., Saberi, A. and Wan, Y.: On Multiple-Delay Static Output Feedback Stabilization of LTI Plants, in American Contr. Conf., Seattle, WA (2008) 419-423.

[27] Tipsuwan, Y. and Chow, M.Y.: Control methodologies in networked control systems, Control Engineering Practice, 11 (2003) 1099-1111.

[28] Yue, D., Han, Q.L. and Peng, C.: State Feedback Controller Design of Networked Control Systems, IEEE Trans. Circuits and Syst. II: Express Briefs, vol. 51, 2004, pp.640-644.

[29] Zhang, W., Branicky, M.S. and Phillip, S.M.: Stability of networked control systems, Contr. Syst. Mag., 21 (2001) 84-99.

\section{APPENDIX}

\section{A1. Chebyshev Polynomials Definitions [17]}

Definition 1: (i) The Chebyshev polynomial $T_{n}(x)$ of the first kind is a polynomial in $x$ of degree $n$, defined by

$$
T_{n}(x)=\cos n \theta \quad \text { when } x=\cos \theta
$$

(ii) The Chebyshev polynomial $U_{n}(x)$ of the second kind is a polynomial in $x$ of degree $n$, defined by

$$
U_{n}(x)=\frac{\sin (n+1) \theta}{\sin \theta} \quad \text { when } x=\cos \theta
$$

\section{A2. LEVERRIER-SAURIAU-Frame Algorithm}

Theorem 2 ([21]): Let the characteristic equation for $A \in$ $\mathbb{R}^{n \times n}$ be given by, $\lambda^{n}+c_{1} \lambda^{n-1}+c_{2} \lambda^{n-2}+\cdots+c_{n}=0$, and define a sequence by taking $B_{0}=I$ and $B_{i}=-\frac{\operatorname{Tr}\left(A B_{i-1}\right)}{i} I+$ $A B_{i-1}$ for $i=1,2, \ldots, n$. Then, the $i$ th coefficient is $c_{i}=$ $-\frac{\operatorname{Tr}\left(A B_{i-1}\right)}{i}$.

\section{A3. Proof of Proposition 2}

Proof: According to Assertion 1(1), the coefficients of $P_{c l}(z)$ satisfy:

$$
a_{\mu}(h, \tau, k)=\sum_{v=1}^{m} k_{v} \alpha_{\mu, v}\left(h, \tau_{v}\right)+\beta_{\mu},
$$

that is,

$$
\frac{\partial a_{\mu}(h, \tau, k)}{\partial k_{v}} \equiv \alpha_{\mu, v}\left(h, \tau_{v}\right)
$$

A straightforward application of Theorem 2 leads to:

$$
\bar{\alpha}_{\mu}(h, \tau)=\alpha_{\mu, v}\left(h, \tau_{\mu}\right),
$$

Assertion 1(2) allows concluding that the above equality is true for all $v$. On the other hand, from Assertion 1(1), $a_{\mu}(h, \tau, 0)=\beta_{\mu}$. Then, a straightforward application of the Induction Method to:

$$
\tilde{\Phi}_{c l}(z, h, \tau, 0)=\left[\begin{array}{cc}
z I-\Phi(h) & 0 \\
e_{1}^{T} & z
\end{array}\right]
$$

shows us that $\beta_{\mu}=\left(\begin{array}{c}n \\ \mu-1\end{array}\right)$. With this fact in mind, we have that $a_{\mu}(h, \tau, k)=(-1)^{n-\mu+1} s_{n-\mu+1}$, taking $\mu=\overline{0, n}$ and putting this in a matrix form we obtain (15). The proof is finished if we show that $A$ is nonsingular. Singularity of $A$ simply means that there exist some dependent row or column vectors. Then, a straightforward application of Assertion 1(2) and Remark 5 implies that $\tau_{1}=\cdots=\tau_{n+1}$. Since, by assumption we have that $\tau_{1}<\cdots<\tau_{n+1}$, the proof is completed. 